ISSN 1997-342X (Online), ISSN 1991-8631 (Print)

Original Paper http://ajol.info/index.php/ijbcs http://indexmedicus.afro.who.int

\title{
Facteurs socioculturels et environnementaux déterminant la connaissance des plantes utilisées pour les soins infantiles au Sud du Bénin
}

\author{
Sabine A. KOUCHADE ${ }^{*}$, C. Aristide. ADOMOU, G. Hospice. DASSOU et \\ Akpovi AKOEGNINOU \\ Laboratoire de Botanique et Ecologie Végétale, Département de Biologie Végétale, Faculté des Sciences et \\ Techniques, Université d'Abomey-Calavi (UAC), 01 BP 4521 Cotonou, Benin. \\ *Auteur correspondant,E-mail : kouchsabine@gmail.com,Tel : (00229) 95281461/97930816
}

\section{RESUME}

$\mathrm{Au}$ Bénin, les plantes médicinales utilisées dans les soins infantiles sont peu documentées et leur connaissance risque de s'éroder progressivement. La présente étude se propose d'identifier les facteurs déterminant les connaissances liées à l'usage de ces plantes au Sud du Bénin. Une enquête ethnobotanique a été réalisée à travers des interviews individuelles et semi-structurées auprès de 708 personnes. Deux analyses de classification basées sur des arbres de décision ont été effectuées. La première a impliqué le nombre de plantes mentionnées par un enquêté et la seconde, le nombre d'usages ou de recettes rapportés. Quatre facteurs : la spécialisation en la médicine traditionnelle, l'appartenance à un groupe socioculturel, la position phytogéographique de l'enquêté et l'origine de la connaissance déterminent la connaissance relative au nombre de plantes utilisées pour traiter les pathologies infantiles. La connaissance relative au nombre de recettes formulées est fonction de la spécialisation en la médecine traditionnelle, de l'appartenance à un groupe socioculturel, et de l'origine de la connaissance. Cette étude pourra contribuer à la préservation du patrimoine végétal, au maintien et au renforcement des connaissances en matière des plantes médicinales infantiles.

(C) 2017 International Formulae Group. All rights reserved.

Mots clés : Plantes médicinales, facteurs socioculturels et environnementaux, pédiatrie traditionnelle, SudBénin.

\section{Socio-cultural and environmental factors determining the knowledge of plants used for child care in southern Benin}

\begin{abstract}
In Benin, medicinal plants used in child care are poorly documented and their knowledge is likely to erode gradually. The present study aimedat identifying the factors determining knowledge related to the use of these plants in southern Benin. An ethnobotanical survey was carried out through semi-structured individual interviews with 708 people. Two classification analyzes based on decision trees were performed. The first involved the number of plants mentioned by each respondent and the second, the number of uses or recipes reported. Factors such as specialization in traditional medicine, ethnicity, phytogeographical position of the respondent and origin of knowledge determine the knowledge related to the number of plants used to treat infantile ailments. Knowledge about the number of recipes formulated depends on the specialization in
\end{abstract}


traditional medicine, ethnicity and the origin of knowledge. This study will contribute to the preservation of the plant heritage, to the maintenance and the strengthening of knowledge in the field of medicinal plants.

(C) 2017 International Formulae Group. All rights reserved.

Keywords: Medicinal plants, sociocultural factors, traditional pediatry, Southern Benin.

\section{INTRODUCTION}

Durant des siècles et même des millénaires, nos ancêtres ont utilisé les plantes pour soulager leurs douleurs, guérir leurs maux et panser leurs blessures (Benkhnigue et al., 2011). L'usage des plantes à des fins thérapeutiques est régi par un environnement socioculturel déterminant l'adoption de la médicine traditionnelle dans les modes de soin de santé des populations locales. Malgré les avancées de la médecine moderne et l'existence de structures de santé, les populations ont toujours recours aux espèces végétales pour leurs soins de santé puisqu'elles gardent encore leur savoir médical souvent hérité des ancêtres, et continuent à l'utiliser. Cependant, la perte des savoirs locaux affecte aussi bien la phytothérapie que sa base, la biodiversité. La diminution $\mathrm{du}$ nombre des personnes détentrices des savoirs présage d'un affaiblissement de la pratique de la médicine traditionnelle (Badiane et al., 2015).

Les études ethnobotaniques permettent non seulement de mettre en valeur les connaissances relatives à l'usage des plantes mais aussi d'assurer la préservation de cette connaissance dans un contexte de modernisation de la vie (Thomas et al., 2009). Ces connaissances liées aux plantes résultent le plus souvent d'une transmission générationnelle, répondant à un ensemble de normes et mécanismes traditionnels dont le côté sacré permet de sauvegarder le secret et, en même temps, d'en limiter la diffusion. Mais, ces connaissances sont en danger à cause de l'introduction des religions réveillées, de l'éducation moderne et du changement de modes de vie (Badiane et al., 2015). A cela s'ajoutent d'autres facteurs dont l'augmentation de l'urbanisation des zones par les populations, la perte d'accès aux ressources traditionnelles, les restrictions des pratiques traditionnelles d'aménagement pour maintenir ces ressources et plus récemment les forces de globalisation et d'industrialisation (Turner et Turner, 2008).

Certaines études ont montré que les connaissances ethnobotaniques sont influencées par le sexe (Mehdiouiet Kahouadji 2007; Benkhnigueet al., 2011), l'âge, le niveau d'étude et la situation matrimoniale des enquêtés (Benkhnigueet al., 2011). Pour d'autres, par contre (Ryan et al., 2008; Dassou et al., 2014; Dassou et al., 2015), l'âge n'influence pas cette connaissance. Ceci indique que les facteurs socioculturels et environnementaux peuvent contribuer à la perte ou à la préservation de la connaissance traditionnelle des plantes. La présente étude a été initiée dans le but de connaître les facteurs socioculturels et environnementaux pouvant agir sur le maintien, le renforcement et la conservation des connaissances ethnobotaniques. Elle a pour objectif général de contribuer à la conservation des connaissances ethnobotaniques liées aux plantes utilisées dans les soins de santé humaine et en particulier ceux des enfants de 0 à 12 ans. De façon spécifique, il s'agit d'identifier et d'analyser les facteurs socioculturels et environnementaux déterminants dans la connaissance des plantes au Sud du Bénin.

\section{MATERIEL ET METHODES \\ Milieu d'étude}

Les études ethnobotaniques ont été conduites à travers tout le Sud du Bénin (Figure 1) qui s'étend entre les latitudes $6^{\circ} 25^{\prime} \mathrm{N}$ et $7^{\circ} 30^{\prime} \mathrm{N}$ et les longitudes $1^{\circ} 35^{\prime} \mathrm{Eet}$ $2^{\circ} 49^{\prime} \mathrm{E}$ et couvre une superficie de 17109 $\mathrm{km}^{2}$. Selon les subdivisions administratives, le Sud du Bénin ou Bénin méridional couvre les 
départements de 1'Atlantique, du Littoral, de l'Ouémé, du Plateau, du Mono, du Couffo et du Zou. Le climat est de type subéquatorial, caractérisé par un régime pluviométrique bimodal avec deux saisons pluvieuses alternées par deux saisons sèches. La température moyenne annuelle est de $28{ }^{\circ} \mathrm{C}$ et l'humidité de l'air varie entre $69 \%$ et $97 \%$ (Akoègninou, 2004). Les sols les plus dominants sont les sols ferralitiques sur sédiments argileux, les sols hydromorphes dans les vallées, les bas-fonds et les plaines alluviales, les vertisols dans la dépression de la Lama et les sols bruns eutrophes tropicaux (Igué et al., 2013).

Sur le plan phytogéographique, le sud du Bénin compte 1170 espèces végétales (Adomou et al., 2011), elle appartient à la zone guinéo-congolaise. La végétation est une mosaïque d'îlots de forêts denses humides, de savanes, de prairies, de mangrove, de champs, de jachères et de plantations diverses (Adomou et al., 2011). Selon le même auteur, la zone d'étude est subdivisée en quatre Districts phytogéographiques (Figure 1): Côtier, Pobè, Vallée de l'Ouémé et Plateau auxquels s'ajoute la partie sud de la zone soudano-guinéenne.

La population est de 5369774 habitants avec une densité qui varie entre 100 habitants $/ \mathrm{km}^{2}$ en général à 322 habitants $/ \mathrm{km}^{2}$ dans le département de l'Atlantique (INSAE, 2013).

\section{Méthodes}

\section{Enquêtes ethnobotaniques}

La population de zone d'étude a été subdivisé en treize (13) groupes ethniques dominants dont les Adja dans le Couffo; les Waci, Gen, Kotafon, Sahouè, Pédah dans le Mono, les Nagot dans le Plateau; les Goun, wémé, Tori dans l'Ouémé ; les Aizo, Fon dans l'Atlantique; les Fon dans les départements du Zou (Figure 1).

Dans chaque aire ethnique, des sites ont été choisis pour l'inventaire des espèces à usages médicinaux selon les critères suivants: l'accessibilité des sites, la célébrité dans la pratique de la médecine traditionnelle, l'usage exclusif ou majoritaire des produits végétaux ou naturels dans le processus de guérison (Dassou et al., 2015). Les individus ont été choisis en tenant compte de leur disponibilité et de leur connaissance en plantes médicinales utilisées dans les soins de santé des enfants. Des enquêtes ont été réalisées par des interviews individuelles et semi-structurées à l'aide de questionnaires. Les différentes rubriques du questionnaire sont: le profil de l'enquêté (sexe, ethnie, âge, niveau d'instruction, taille de ménage, spécialité dans l'utilisation de la médecine traditionnelle, etc.), les noms des maladies des enfants traitées, les noms locaux des espèces végétales, les organes végétaux utilisés, les recettes y afférentes, l'origine de la connaissance (parents, amis, etc.). Les plantes citées ont été récoltées ou achetées et herborisées. L'identification botanique a été réalisée à l'Herbier National du Bénin par comparaison avec les herbiers de référence ou avec la flore analytique du Bénin (Akoègninou et al., 2006).

\section{Traitement des données}

Les facteurs qui influencent la connaissance liée aux plantes utilisées dans le traitement des maladies infantiles au Sud du Bénin ont été déterminés à partir de deux analyses de classification basées sur des arbres de décision (Banerjee et al., 2014). La première a impliqué le nombre de plantes mentionnées par un enquêté et la seconde, le nombre d'usages ou de recettes rapportés. L'utilisation des arbres de décision repose sur la répartition des individus suivant les valeurs d'un certain nombre d'attribut. Il s'agit d'une méthode dite de partitionnement récursif des données. En effet, la méthode construit des classes d'individus les plus homogènes possibles en posant une succession de questions binaires (de type oui/non) sur les attributs de chaque individu. Les arbres de décision fournissent une représentation graphique qui est sous forme d'un arbre constitué de feuilles terminales (les classes d'individus) obtenues en suivant un chemin le long des nœuds, chaque nœud correspond à 
une question binaire utilisant une variable de jeu de données. Les arbres de décision permettent donc d'identifier très rapidement les variables les plus discriminantes d'un jeu de données en fonction de leur présence parfois répétée le long des nœuds (Banerjee et al., 2014).

Les analyses ont impliqué 13 variables dont 03 sont dépendantes ou expliquées et 10 indépendantes ou explicatives (Tableau 1). La connaissance a été évaluée par rapport au nombre de plantes mentionnées par un individu, au nombre total d'usages ou nombre de recettes rapportés par un individu et au nombre de vertus thérapeutiques ou nombre de maladies par un enquêté.

Un traitement basique de jeu de données par arbre de décision construit avec l'algorithme CART (pouvant fonctionner avec tous types de variables : qualitatives, ordinales et quantitatives continues) avec le logiciel R.

Un test de corrélation a été réalisé pour étudier le lien entre le nombre de recettes rapportées et le nombre de plantes mentionnées par chaque individu.

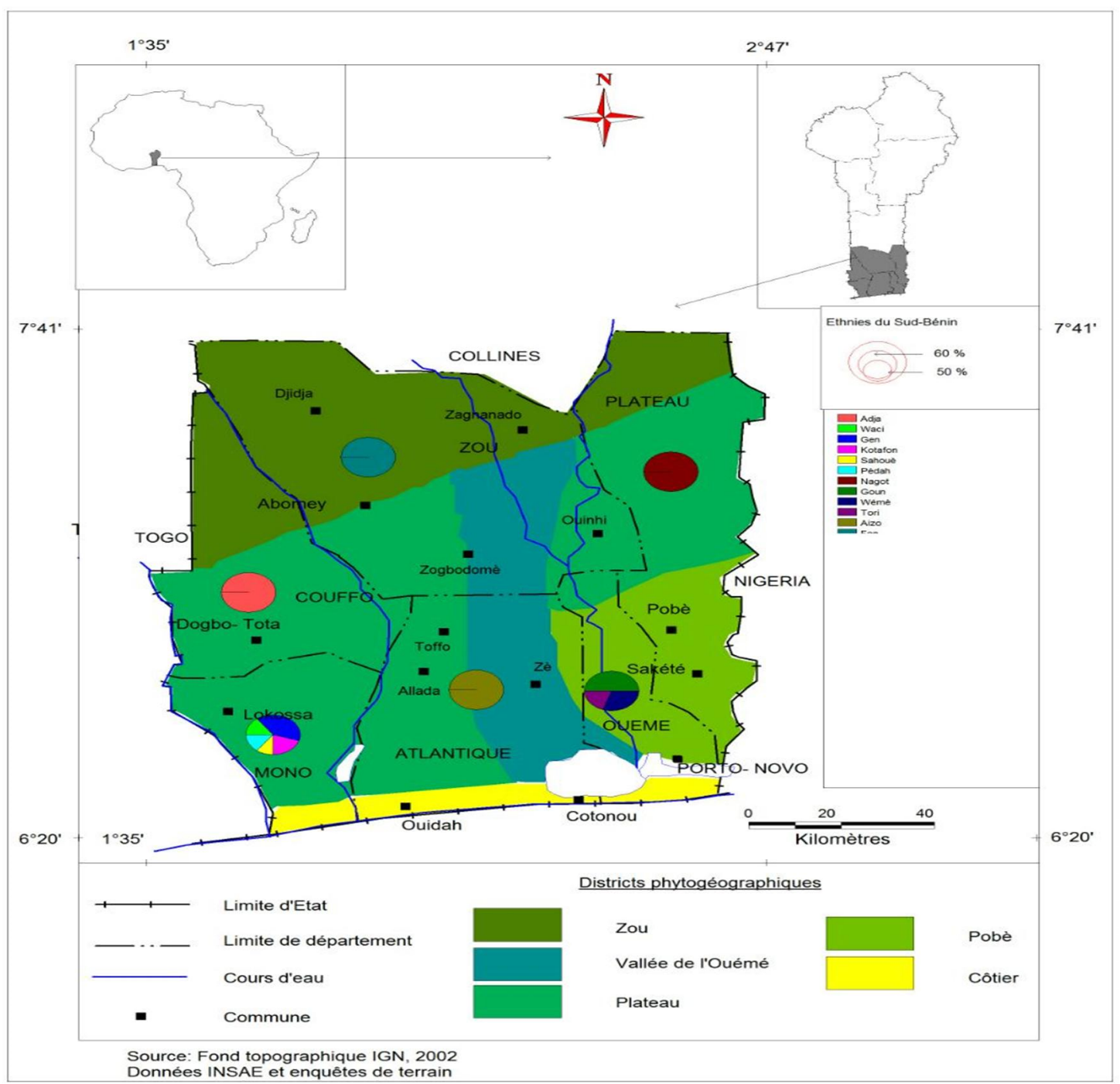

Figure 1 : Carte de la zone montrant les ethnies et les districts phytogéographiques. 
Tableau 1 : Description des 13 variables impliquées dans l'arbre de décision.

\begin{tabular}{lccc}
\hline Variables & Codes & Signification & Types de \\
variables
\end{tabular}

\section{RESULTATS}

Catégorisation des variables déterminant la connaissance en ethnomédecine infantile

CART a identifié 4 déterminants

(Nodes) dont la spécialisation (Node 1) comme le principal facteur expliquant la variabilité de la connaissance relative au nombre de plantes citées par la population (n $=708$ ) pour traiter les maladies infantiles au
Sud-Bénin (Figure 2). La première branche ou arête de l'arbre (à gauche), constituant la première sous-population, rassemble les individus qui ne sont pas déclarés spécialistes de la médecine traditionnelle infantile (Auc) et ceux reconnus comme tradipraticiens (Tra) dans la zone d'étude. Ces deux types d'individus ont édifié le nœud 2 (Node $2 ; n=$ 
$650)$ et ont cité en moyenne 5 plantes médicinales.

La fragmentation du nœud 2, qui est fonction de l'ethnie, a généré deux classes de groupes ethniques dont la plus importante regroupe 7 groupes ethniques à savoir : Adja, Fon, Goun, Holli, Nago, Tori et Wémè (Node $3 ; \mathrm{n}=352$ ) avec un nombre moyen de 9 plantes citées. Le second groupe d'ethnies, constitué des Aïzo, Cotafon, Mina, Pédah, Sahouè et Wachi et formant le nœud 14 ( $\mathrm{n}=$ 298) n'a cité qu'en moyenne 3 plantes. Dans ce groupe, la spécialisation permet de séparer les ethnies non spécialistes de la médecine traditionnelle infantile (en moyenne 5 plantes) de celles des spécialistes des pratiques traditionnelles ( 7 plantes en moyenne). Pour appréhender le niveau de connaissance, la classe d'ethnies majoritaire (Node $3 ; \mathrm{n}=352$ ) a subi deux partitionnements successifs par le biais de deux facteurs. Premièrement, la position phytogéographique (Nodes 4 et 9) a séparé les Adja, Wémè et Fon situées dans la Vallée de l'Ouémé et sur le Plateau, les Holli, Nagot, Tori, Goun et Fon réparties dans les districts de Pobè et du Zou. Secondairement, l'origine de la connaissance (Nodes 5 et 11) a facilité la segmentation finale. Ainsi, 6 feuilles pures (nœuds terminaux) sont obtenues à partir de la classe d'ethnies majoritaire dont les descripteurs importants sont: l'ethnie associée d'une part au positionnement phytogéographique des populations (Nodes 7 et 10 avec respectivement 14 et 7 plantes citées en moyenne) et d'autre part à l'origine de la connaissance (Node 6; en moyenne 12 plantes citées).

La seconde arête de l'arbre, à droite, a porté les vendeurs de plantes médicinales qui ont cité en moyenne 24 plantes médicinales. Elle a assuré la création du nœud $17(\mathrm{n}=58)$ qui a subi un partitionnement par le biais de la variable zone phytogéographique pour produire 2 feuilles pures. Les unes sont constituées des vendeurs et vendeuses situés dans les districts côtier et Zou (en moyenne 32 plantes citées) et les autres sont composées des vendeurs et vendeuses localisés dans les districts Vallée de l'Ouémé, Pobè et Plateau (en moyenne 19 plantes citées).

$\mathrm{Au}$ total, 5 décisions ou conclusions peuvent être tirées de cet arbre :

Quatre facteurs (la spécialisation, l'ethnie, la position phytogéographique et l'origine de la connaissance) déterminent la connaissance relative au nombre de plantes utilisées pour traiter les pathologies infantiles au Sud-Bénin;

Le nombre de plantes connues pour traiter les pathologies infantiles décroît des vendeurs et vendeuses de plantes médicinales aux non spécialistes de médecine infantile en passant par les tradipraticiens ;

Les ethnies à savoir: Adja, Fon, Gun, Holli, Nago, Tori et Wémè connaissent plus de plantes médicinales à usages infantiles que les ethnies : Aïzo, Cotafon, Mina, Pédah, Sahouè et Wachi ;

La connaissance de plantes médicinales à usages infantiles est plus élevée chez les vendeurs et vendeuses situés dans les districts côtier et Zou que chez ceux localisés dans les districts Vallée de l'Ouémé, Pobè et Plateau ;

Les individus ayant reçu une formation en ethnomédecine détiennent une connaissance plus aigüe et approfondie des plantes utilisées.

Un CART à partir du nombre de recettes rapportées par les 708 personnes enquêtées pour traiter les maladies infantiles produit un arbre avec 3 variables comme critères de partitionnement. Par ordre d'importance, il y a : l'ethnie, la spécialisation et l'origine de la connaissance (Figure 2). Deux grands groupes d'ethnies ont été isolés. Le premier, le plus important, porté par la branche gauche de l'arbre, regroupe les Adja, Fon, Holli, Nagot, Tori et Wémè et forme le nœud 2 (Node 2, $\mathrm{n}=342$ ). Les Adja et Nagot (Node 6), non déclarés spécialistes de la médecine traditionnelle infantile (Auc), ont cité moins de recettes (4 recettes en moyenne ; Node $7 ; \mathrm{n}=89$, err $=166,5)$ que ceux reconnus comme tradipraticiens (Tra) dans la 
zone d'étude ( 7 recettes en moyenne; Node $8 ; \mathrm{n}=8$, err $=84)$. Les Fon ont rapporté, quant à eux, en moyenne 8 recettes (Node 9 ; $\mathrm{n}=10$, err $=176,4)$. Malgré la similitude, CART a indiqué qu'il existe une certaine variabilité entre les nombres de recettes rapportées par les 6 ethnies du premier groupe. Ce qui est à l'origine des partitionnements enregistrés au niveau des nœuds 2 et 3 . Ainsi, les individus, non déclarés spécialistes (Auc) et ceux reconnus comme tradipraticiens (Tra), appartenant aux ethnies Adja, Fon, Holli, Nagot, Tori et Wémè sont séparés des vendeurs et vendeuses de ces mêmes ethnies qui ont rapporté plus de recettes (Node $12 ; 9$ recettes en moyenne; $n$ $=35$, err $=1379$ ). De plus, les Adja, Fon, Nagot et Tori sont organisés en deux classes : les individus (Node 5; $\mathrm{n}=107$ ) dont la connaissance provient des enseignements au cours des formations en ethnomédecine (Eco) et d'un héritage (Her) et ceux ayant bénéficié d'un bain de connaissances auprès des vendeurs de plantes au cours des achats de plantes médicinales (Node $10 ; 4$ recettes en moyenne $; \mathrm{n}=98$, err $=231,6)$.

Le groupe d'ethnies porté par la branche droite de l'arbre, est constitué de Aïzo, Cotafon, Goun, Mina, Pédah, Sahouè et Wachi (Node 13; $\mathrm{n}=366$ ). Son partitionnement sous l'influence de la spécialisation a généré 4 feuilles pures dont la plus importante est le nœud 19 qui regroupe les vendeurs et vendeuses de plantes médicinales de ces 7 ethnies (Node 19; en moyenne 7 recettes rapportées; $\mathrm{n}=23$; err $=$ 286,6). Les individus des autres feuilles ont rapporté successivement 3 recettes (Node 15 ; $\mathrm{n}=194$, err $=268,7) ; 2($ Node $17 ; \mathrm{n}=141 ;$ err $=118,9)$ et 4 recettes (Node $18 ; \mathrm{n}=8$; err $=20$ ).

$\mathrm{Au}$ total, les décisions suivantes peuvent être tirées de l'arbre :

la connaissance relative au nombre de recettes formulées est fonction de 3 variables à savoir : l'ethnie, la spécialisation de l'individu dans la médecine traditionnelle et l'origine de la connaissance ;

les ethnies Adja, Fon, Holli, Nagot, Tori et Wémè connaissent plus de recettes que les ethnies Aïzo, Cotafon, Gun, Mina, Pédah, Sahouè et Wachi ;

les vendeurs et vendeuses de plantes connaissent plus de recettes que les non spécialistes et tradipraticiens ;

l'héritage et les formations sur l'ethnomédecine sont des sources plus pointues et favorables à la connaissance en ethnomédecine infantile.

\section{Déterminants socioculturels et environnementaux et connaissance en ethnomédecine infantile}

L'échantillon est majoritairement représenté par l'ethnie Fon avec un taux de $11 \%$ (Tableau 2). La moyenne de plantes citées par individu de la population est de 7 plantes. Elle est plus élevée chez les Fon et Goun (10 plantes en moyenne chacun) et varie de façon significative d'une ethnie à l'autre $(\mathrm{F}$ $=10,47 ; \mathrm{p}=0 ; \mathrm{ddl}=12$ ). Le nombre moyen de recettes rapportées par individu équivaut à 4 recettes par individu enquêté. Il varie de 2 pour les Pédah et Wachi à 7 pour les Holli. Les résultats d'analyse de variance montrent une différence très hautement significative ( $p$ $=0$ ) entre les nombres moyens de recettes rapportées par les ethnies ( $\mathrm{F}=18,54 ; \mathrm{p}=0$; $\mathrm{ddl}=12$ ).

En considérant le sexe, les enquêtés ont été en majorité des femmes (63\%). Les valeurs moyennes de plantes citées varient de façon très hautement significative en fonction du sexe $(F=20,44 ; p=0 ; d d l=1)$. Elles sont comprises entre 5 pour les hommes et 8 pour les femmes. Quant à la moyenne du nombre de recettes rapportées, les valeurs variaient significativement $(\mathrm{F}=3,83 ; \mathrm{p}=$ $0,05 ; \mathrm{ddl}=1)$ de 3 pour les hommes à 5 pour les femmes. 
La plupart des enquêtés (62\%) sont non instruits. Néanmoins, la moyenne du nombre de plantes citées est similaire d'un niveau d'instruction à un autre $(\mathrm{F}=2,32 ; \mathrm{p}=0,07$; $\mathrm{ddl}=3$ ). L'observation contraire est faite pour le nombre moyen de recettes rapportées qui a montré une variation significative en fonction du niveau d'instruction $(\mathrm{F}=3,53 ; \mathrm{p}=0,01$, $\mathrm{ddl}=3$ ). Il est plus faible pour les trois premiers niveaux ( 4 recettes chacun) et a atteint sa valeur la plus élevée (6 recettes) pour le niveau universitaire.

L'âge des enquêtés varie de 21 à 83 ans avec une moyenne de 46 ans pour la population. Les individus d'âge compris entre 40 à 59 ans sont majoritaires avec un taux de $64 \%$. Le nombre moyen de plantes citées varie de 5 pour les vieux à 7 pour les adultes mais cette variation enregistrée n'est pas significative $(F=1,17 ; p=0,31 ; d d l=2) . \mathrm{La}$ moyenne de recettes rapportées varie significativement de 3 pour les jeunes et vieux à 5 pour les adultes $(\mathrm{F}=3,38 ; \mathrm{p}=0,03 ; \mathrm{ddl}=$ 2).

Concernant la profession, la majorité de la population (32\%) est des agriculteurs. Cependant, le nombre moyen de plantes citées est plus élevée chez les revendeurs ( 9 plantes). Sa valeur minimale (2 plantes) est obtenue pour les pêcheurs. De plus, la variation observée pour ce paramètre était significative $(F=4,45 ; p=0 ; d d l=7)$. En ce qui concerne le nombre moyen de recettes rapportées, il a montré aussi une variation significative $(\mathrm{F}=$ 2,$92 ; \mathrm{p}=0,005 ; \mathrm{ddl}=7$ ) en fonction de la profession. Il est compris entre 2 pour les pêcheurs et 7 pour les fonctionnaires retraités.

La médecine traditionnelle infantile n'est pas une activité principale pour la plupart des enquêtés (85\%). Seulement $8 \%$ des enquêtés sont herboristes et $7 \%$ des guérisseurs. Pourtant, le nombre moyen de plantes mentionnées le plus élevé (24 plantes) est obtenu pour les herboristes. Les valeurs obtenues pour ce paramètre varient très significativement $(\mathrm{F}=346,70 ; \mathrm{p}=0 ; \mathrm{ddl}=2)$ en fonction de la spécialisation. Par rapport à la moyenne du nombre de recettes, les valeurs sont comprises entre 3 pour les non spécialistes de la médecine traditionnelle et 8 pour les herboristes. Les variations entre ces valeurs ont montré une différence significative $\mathrm{F}=79,07 ; \mathrm{p}=0 ; \mathrm{ddl}=2$ ) en fonction de la spécialisation.

La connaissance détenue par les populations dans le traitement des maladies infantiles provient globalement de trois sources. Soixante-quatre pourcent $(64 \%)$ des personnes enquêtées ont hérité leur connaissance de leurs parents (transmission verticale). En effet, le nombre moyen de plantes mentionnées est plus faible (4 plantes) chez les individus ayant bénéficié d'un bain de savoir au cours des achats de plantes médicinales et a atteint sa valeur élevée (11 plantes) chez ceux qui ont participé à des formations sur la médecine traditionnelle. Au regard du nombre moyen de recettes, l'état de connaissance est le même à savoir que le niveau de connaissance crôit des individus dont le savoir est issu des achats (3 recettes) à ceux qui ont subi des formations (6 recettes).

En considérant la religion, l'échantillon est majoritairement représenté par les enquêtés pratiquant les religions endogènes (les animistes) (51\%). Quel que soit le paramètre (moyennes de plantes et de recettes), la connaissance varie significativement en fonction de la religion (respectivement $\mathrm{F}=4,87 ; \mathrm{p}=0,001 ; \mathrm{ddl}=4$ et $F=2,54 ; p=0,03 ; d d l=4)$. Les enquêtés pratiquant les religions endogènes connaissent plus les plantes ( 9 plantes citées) alors que ce sont les célestes qui ont formulé plus de recettes (6 recettes rapportées) (Tableau 2).

Pour l'ensemble de la population, le nombre moyen d'années d'expérience dans l'utilisation des espèces végétales pour les soins de santé des enfants est de 22 ans. Trente-cinq pourcent (35\%) d'eux ont entre 
10 et 20 ans d'expérience. Les valeurs du nombre de plantes citées ne varient pas significativement $(\mathrm{F}=0,13 ; \mathrm{p}=1,51 ; \mathrm{ddl}=$ 6) en fonction de la classe d'années d'expérience. Cependant, la valeur la plus élevée du nombre de plantes citées ( 8 plantes) est obtenue pour les individus ayant une année d'expérience comprise entre 30 et 39 ans. Au regard du nombre de recettes rapportées, la différence est significative $(\mathrm{F}=3,28 ; \mathrm{p}=$ $0,003 ; \mathrm{ddl}=6)$. En effet, le nombre moyen de recettes rapportées varie de 3 pour les individus ayant entre 1 et 9 ans d'expérience à 5 pour ceux qui ont entre 30 et 59 ans.

Quarante-six pourcent (46\%) des enquêtés sont localisés dans le district phytogéographique du Plateau. Les populations du district Zou ont cité en moyenne plus de plantes (10) que les autres.
Les résultats d'analyse de variance ont montré qu'il existe une différence hautement significative $(F=16,51 ; p=0 ; d d l=4)$ entre cette valeur et celles obtenues pour les autres districts. Les observations similaires ont été faites par rapport à la variation du nombre de recettes en fonction de la localisation phytogéographique $(\mathrm{F}=26,19 ; \mathrm{p}=0$; ddl $=$ 4). Les valeurs varient entre 3 pour le district du Plateau et 6 pour les districts de Vallée de l'Ouémé et du Zou.

Globalement, dans la population étudiée, le nombre de recettes rapportées évolue positivement $(r=0,73)$ et de façon très hautement significative $(\mathrm{p}=0)$ avec le nombre de plantes citées. La variation du nombre de recettes s'explique par $53,6 \%$ de la variation du nombre de plantes mentionnées selon la valeur de $\mathrm{R}^{2}$ de la fonction de régression.

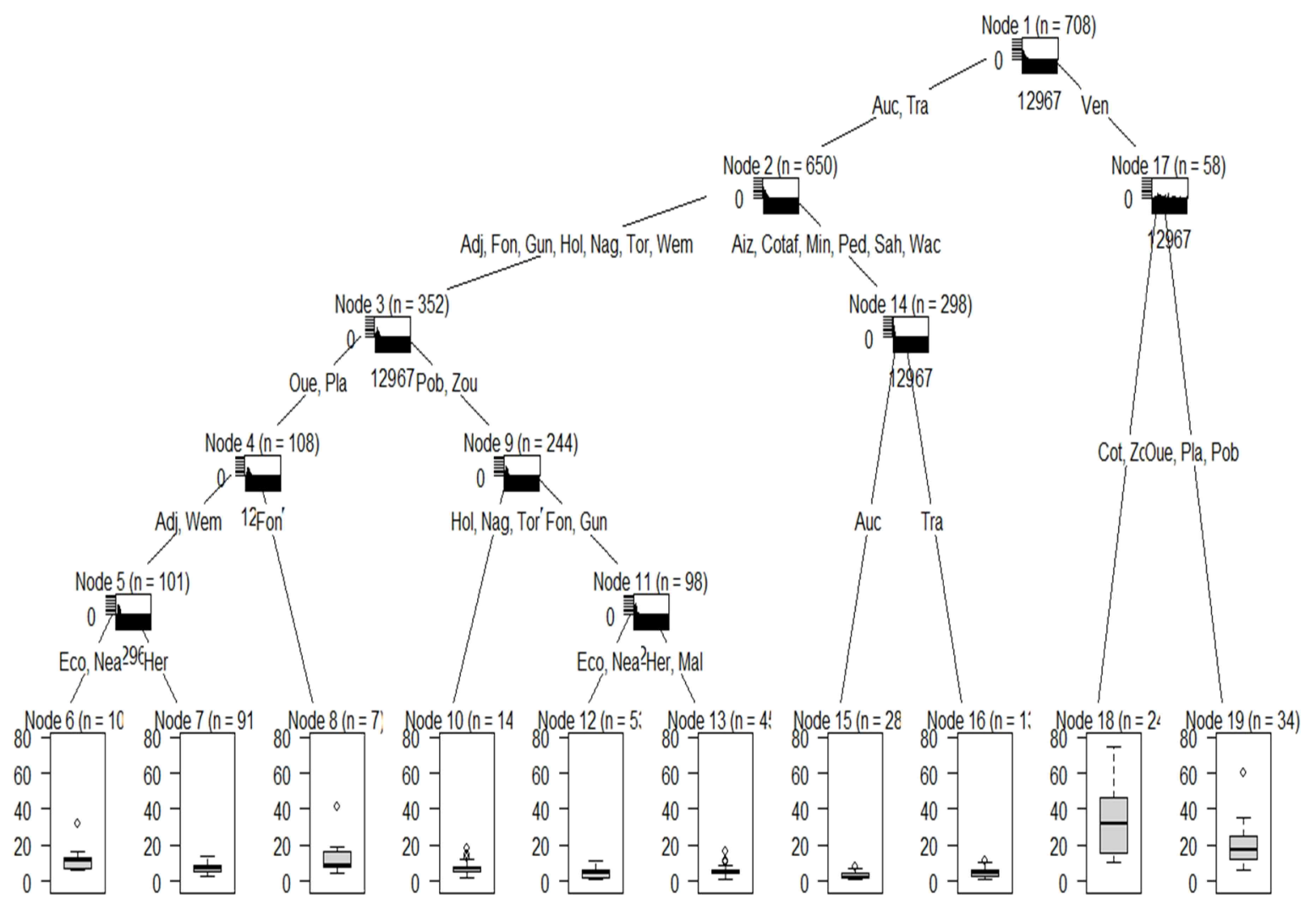

Figure 2 : Arbre de décision décrivant la variabilité du nombre de plantes. 
Tableau 2: Récapitulatif des moyennes des nombres de plantes et de recettes selon les caractéristiques socioculturelles et environnementales des enquêtées.

\begin{tabular}{|c|c|c|c|c|}
\hline Paramètres & $\begin{array}{c}\text { Nombre } \\
\text { d'individus }\end{array}$ & Pourcentage (\%) & $\begin{array}{c}\text { Moyenne N. } \\
\text { de plantes } \\
\text { citées }\end{array}$ & $\begin{array}{c}\text { Moyenne } \mathbf{N} \text {. } \\
\text { de recettes } \\
\text { citées }\end{array}$ \\
\hline \multicolumn{5}{|l|}{ Appartenance sociolinguistique } \\
\hline Adja & 55 & 8 & $7 \pm 4$ & $5 \pm 2$ \\
\hline Aizo & 58 & 8 & $4 \pm 5$ & $3 \pm 3$ \\
\hline Cotafon & 51 & 7 & $4 \pm 5$ & $3 \pm 2$ \\
\hline Fon & 74 & 11 & $10 \pm 13$ & $6 \pm 5$ \\
\hline Goun & 58 & 8 & $10 \pm 13$ & $4 \pm 2$ \\
\hline Holli & 50 & 7 & $8 \pm 3$ & $7 \pm 3$ \\
\hline Mina & 52 & 7 & $3 \pm 2$ & $3 \pm 1$ \\
\hline Nagot & 54 & 8 & $8 \pm 6$ & $5 \pm 2$ \\
\hline Pedah & 50 & 7 & $3 \pm 2$ & $2 \pm 1$ \\
\hline Sahouè & 52 & 7 & $3 \pm 2$ & $3 \pm 1$ \\
\hline Tori & 54 & 8 & $9 \pm 9$ & $4 \pm 1$ \\
\hline Watchi & 50 & 7 & $3 \pm 1$ & $2 \pm 1$ \\
\hline Wémè & 50 & 7 & $9 \pm 4$ & $6 \pm 4$ \\
\hline \multicolumn{5}{|l|}{ Sexe } \\
\hline Masculin & 259 & 37 & $5 \pm 4$ & $3 \pm 3$ \\
\hline Féminin & 449 & 63 & $8 \pm 9$ & $5 \pm 3$ \\
\hline \multicolumn{5}{|l|}{ Niveau d'instruction } \\
\hline Non instruit (N0) & 440 & 62 & $7 \pm 8$ & $4 \pm 3$ \\
\hline Niveau primaire (N1) & 171 & 24 & $6 \pm 6$ & $4 \pm 2$ \\
\hline Niveau secondaire (N2) & 91 & 13 & $6 \pm 6$ & $4 \pm 3$ \\
\hline Niveau universitaire (N3) & 6 & 1 & $7 \pm 4$ & $6 \pm 4$ \\
\hline \multicolumn{5}{|l|}{ Classe d'âge } \\
\hline Jeune (âge $\leq 39$ ans) & 188 & 27 & $6 \pm 8$ & $3 \pm 2$ \\
\hline Adulte (40 ans $\leq$ âge $\leq 59$ ans) & 452 & 64 & $7 \pm 8$ & $5 \pm 2$ \\
\hline Vieux (âge $\geq 60$ ans) & 68 & 9 & $5 \pm 4$ & $3 \pm 3$ \\
\hline \multicolumn{5}{|c|}{ Nombre d'années d'expériences dans l'utilisation des plantes (en ans) } \\
\hline 1 à 9 & 79 & 11 & $6 \pm 8$ & $3 \pm 2$ \\
\hline 10 à 19 & 235 & 33 & $7 \pm 8$ & $4 \pm 3$ \\
\hline 20 à 29 & 229 & 32 & $6 \pm 5$ & $4 \pm 2$ \\
\hline 30 à 39 & 102 & 14,9 & $8 \pm 10$ & $5 \pm 4$ \\
\hline 40 à 49 & 46 & 7 & $6 \pm 4$ & $5 \pm 3$ \\
\hline
\end{tabular}




\begin{tabular}{|c|c|c|c|c|}
\hline Paramètres & $\begin{array}{c}\text { Nombre } \\
\text { d'individus }\end{array}$ & Pourcentage (\%) & $\begin{array}{c}\text { Moyenne N. } \\
\text { de plantes } \\
\text { citées }\end{array}$ & $\begin{array}{c}\text { Moyenne N. } \\
\text { de recettes } \\
\text { citées }\end{array}$ \\
\hline 50 à 59 & 16 & 2 & $7 \pm 6$ & $5 \pm 3$ \\
\hline 60 & 1 & 0,1 & 6 & 4 \\
\hline \multicolumn{5}{|c|}{ Spécialisation dans la médicine traditionnelle } \\
\hline Aucune spécialisation & 602 & 85 & $5 \pm 3$ & $3 \pm 2$ \\
\hline Herboriste & 58 & 8 & $24 \pm 15$ & $8 \pm 5$ \\
\hline Tradipraticien & 48 & 7 & $7 \pm 4$ & $5 \pm 3$ \\
\hline \multicolumn{5}{|l|}{ Professions principales } \\
\hline Artisan & 116 & 16 & $5 \pm 4$ & $4 \pm 3$ \\
\hline Cultivateur & 179 & 25 & $5 \pm 3$ & $4 \pm 3$ \\
\hline Ménagère & 251 & 35 & $8 \pm 9$ & $4 \pm 2$ \\
\hline Pêcheur & 12 & 2 & $2 \pm 1$ & $2 \pm 1$ \\
\hline Revendeur & 108 & 15 & $9 \pm 11$ & $5 \pm 5$ \\
\hline Chasseur & 7 & 1 & $6 \pm 2$ & $5 \pm 2$ \\
\hline Salarié & 31 & 4 & $7 \pm 7$ & $5 \pm 4$ \\
\hline Retraité & 4 & 1 & $8 \pm 5$ & $7 \pm 3$ \\
\hline \multicolumn{5}{|l|}{ Origine de la connaissance } \\
\hline Héritage & 455 & 64 & $8 \pm 8$ & $4 \pm 3$ \\
\hline Connaissance issue des achats & 243 & 34 & $4 \pm 3$ & $3 \pm 2$ \\
\hline Connaissance issue d'une formation & 10 & 2 & $11 \pm 9$ & $6 \pm 4$ \\
\hline \multicolumn{5}{|l|}{ Religions } \\
\hline Endogènes & 359 & 51 & $9 \pm 11$ & $4 \pm 2$ \\
\hline Catholique & 170 & 24 & $8 \pm 8$ & $4 \pm 3$ \\
\hline Evangélique & 120 & 17 & $6 \pm 6$ & $4 \pm 3$ \\
\hline Céleste & 28 & 4 & $8 \pm 8$ & $6 \pm 4$ \\
\hline Musulmane & 31 & 4 & $5 \pm 6$ & $4 \pm 3$ \\
\hline \multicolumn{5}{|l|}{ Districts phytogéographiques } \\
\hline Côtier & 59 & 8 & $8 \pm 13$ & $4 \pm 3$ \\
\hline Plateau & 316 & 45 & $4 \pm 4$ & $3 \pm 2$ \\
\hline Pobè & 212 & 30 & $8 \pm 6$ & $5 \pm 2$ \\
\hline Vallée de l'Ouémé & 53 & 7 & $9 \pm 4$ & $6 \pm 4$ \\
\hline Zou & 68 & 10 & $10 \pm 13$ & $6 \pm 6$ \\
\hline
\end{tabular}




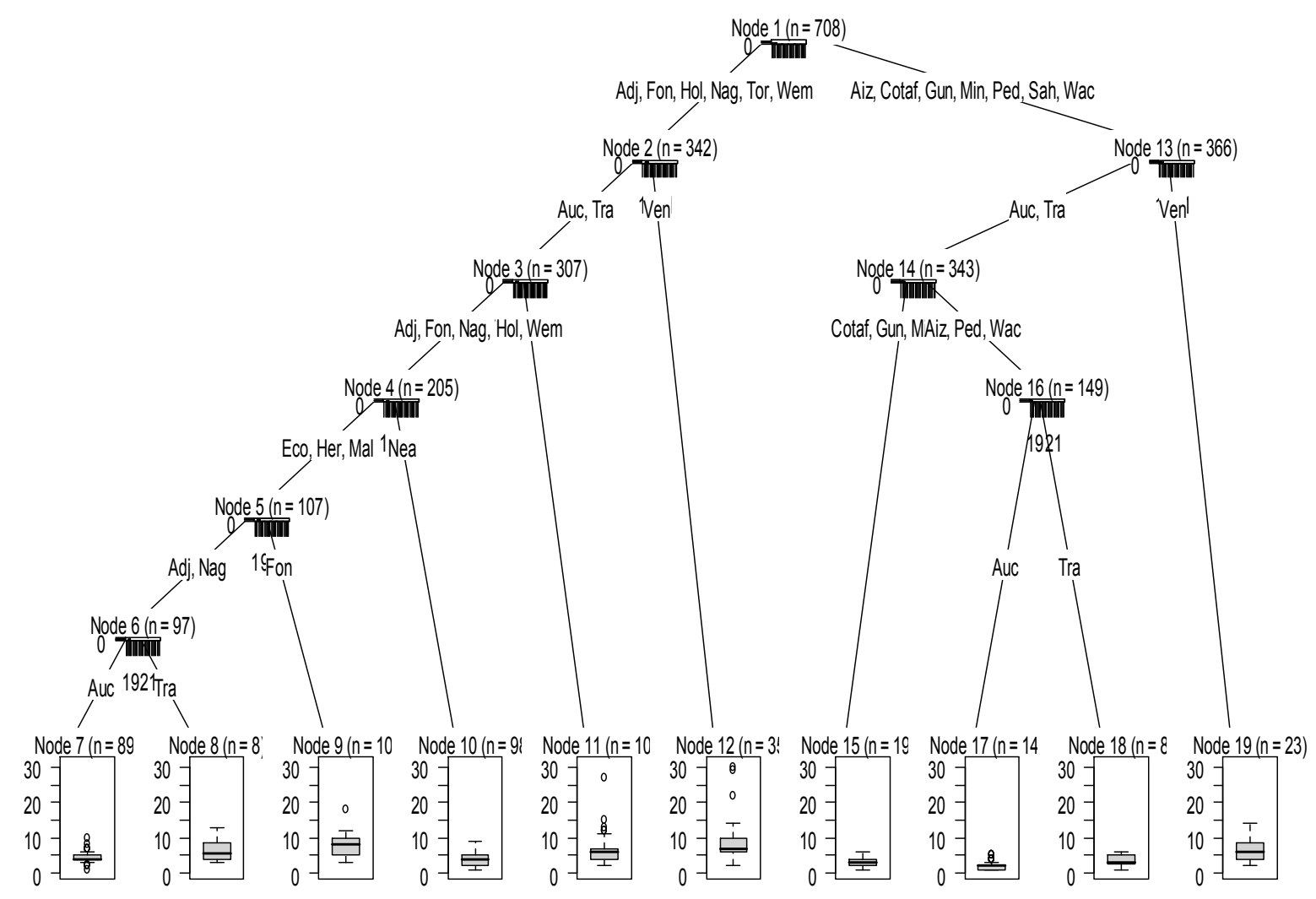

Figure 3 : Arbre de décision décrivant la variabilité du nombre de recettes rapportées.

\section{DISCUSSION}

Les connaissances relatives au nombre de plantes utilisées par les populations pour traiter les maladies infantiles au Sud du Bénin sont fonction des variables : l'appartenance à un groupe socioculturel (ethnie), la spécialisation dans la médecine traditionnelle, l'origine de la connaissance et la position phytogéographiques des enquêtés. Celles relatives au nombre de recettes formulées sont fonction des variables: l'appartenance à un groupe socioculturel (ethnie), la spécialisation dans la médecine traditionnelle et l'origine de la connaissance. Ces résultats contrastent avec ceux Lougbégnon et al. (2011) et ceux de Gouwakinnou et al. (2011); Assogbadjo et al. (2011). En effet, Lougbégnon et al. (2011) ont montré que les connaissances ethnobotaniques liées aux espèces de la réserve forestière marécageuse de la vallée de Sitatunga et zones connexes varient avec l'âge et le sexe. Les travaux de Gouwakinnou et al. (2011) et ceux de Assogbadjo et al. (2011) ont révélé que les connaissances ethnobotaniques liées aux espèces sont fonction de l'âge, du sexe et du groupe ethnique. Mais Dassou et al. (2015) ont indiqué que les zones agroécologiques et phytogéographiques, l'ethnie et le niveau d'instruction constituent les déterminants de la connaissance en médecine vétérinaire traditionnelle au Bénin.Or, de façon générale, les connaissances ethnobotaniques selon les travaux de Byg and Balev (2004) et de Beltràn-Rodriguez et al. (2014), sont globalement influencées par les facteurs dont l'ethnie, l'âge, le sexe, la religion, le niveau d'instruction, la position géographique et la profession de l'enquêté.

La variation des connaissances ethnobotaniques serait également due au fait 
que lors des enquêtes, les enquêtés pourraient oublier les maladies et/ou les plantes utilisées dans le traitement des maladies et s'en rappeler plus tard.

Une ethnie ou un groupe socio-culturel est un ensemble humain constitué par une communauté de langues, de culture, de structures sociales et économiques. Au Bénin, les ethnies diffèrent par leurs habitudes cultuelles, leur savoir-faire, leur savoir-vivre qu'elles se transmettent de génération en génération (Donou Hounsode et al., 2016). On observe donc des différences dans la perception et l'utilisation des ressources phyto génétiques selon les ethnies. Ce qui prouve que certaines ethnies sont plus portées vers l'utilisation des plantes médicinales que d'autres, et justifierait les résultats de cette étude qui stipulent que les ethnies Adja, Fon, Goun, Holli, Nagot, Tori et Wémè connaissent plus de plantes et de recettes pour traiter les maladies infantiles que les autres ethnies. De plus, le nombre de plantes citées par les ethnies Fon et Goun (en moyenne 10 pour les deux ethnies) est plus élevé que ceux cités par les autres ethnies mais l'ethnie Holli a cité plus de recettes ( 7 recettes en moyenne). Ces résultats indiquent que la médecine traditionnelle est un symbole de l'identité culturelle (Yoro, 2012 ; Zerbo et al., 2011) et la connaissance liée à elle se transmet de génération en génération au sein de la société ou de la famille (Dassou et al.,2015 ; Zerbo et al., 2011) ; c'est donc un héritage culturel.

Par ailleurs, les travaux de Kouchadé et al. (2016) sur les plantes médicinales infantiles vendues dans les marchés au sud du Bénin ont permis de savoir que les vendeurs et vendeuses (herboristes) de plantes médicinales les plus représentés appartiennent aux groupes socioculturels Fon et Goun. Or, ces herboristes sont des individus qui capitalisent beaucoup d'informations sur les plantes. En effet, ils sont en contact avec les fournisseurs (c'est-à-dire les grossistes ou les récolteurs de plantes médicinales), d'autres groupes de thérapeutes (guérisseur, chasseur, devins, voyants, etc.), les malades et autres usagers avec lesquels des échanges de connaissances se font régulièrement. Ceci justifie le fait qu'en tenant compte de la spécialisation dans la médecine traditionnelle, les herboristes connaissent plus de plantes et de recettes que les tradipraticiens et les non spécialistes (les parents des enfants). Ils sont, au fait, des individus qui vivent de la vente des produits végétaux, connaissent l'utilité médicinale des plantes et sont aussi soucieux de la sauvegarde de leur patrimoine culturel (Traore, 2015). Selon le même auteur, les herboristes sont spécialistes de la pédiatrie dans le secteur traditionnel, et traitent les maladies infantiles telles que la fièvre, le paludisme, les maux de tête, les maux de ventre, la poussée de dents, les diarrhées, les infections ORL (l'Oto-Rhino-Laryngologie), etc. Ainsi, les herboristes, de par leur savoirfaire, leur savoir-être et leur proximité avec les plantes, mettent à la portée de la population des soins plus accessibles aux plans socio-économique et culturel.

Pour les tradipraticiens ou les guérisseurs, le domaine de la médecine traditionnelle est celui où le secret est recommandé (Yoro, 2012), car pour ces derniers, ce savoir ne doit être transmis qu'aux initiés. A ce propos, les guérisseurs hésitent longtemps et manifestent une certaine réticence lors des enquêtes. Aussi, la réticence constatée peut s'expliquer par le fait que ces derniers tirent leurs ressources financières de la vente de ces recettes. Ceci justifierait la faible connaissance du nombre de plantes $(7$ plantes) citées par ces derniers contre 24 plantes citées par les herboristes.

S'agissant de la position géographique, la connaissance de plantes médicinales à usages infantiles est plus élevée chez les herboristes situés dans les districts phytogéographiques côtier et Zou que chez ceux localisés dans les districts phytogéographique Pobè, Plateau et la Vallée de l'Ouémé. Les districts phytogéographiques représentent des écosystèmes ou des habitats pour certains organismes vivants ou pour certaines plantes en particulier (Sinsin et Kampmann, 2010). Dans ces écosystèmes, règnent des conditions édaphoclimatiques et 
géomorphologiques homogènes qui déterminent la répartition des peuples sur l'ensemble du territoire national (Sinsin et Kampmann, 2010). Ainsi, dans un milieu occupé par un peuple, la diversité floristique est fonction de la zone écologique qu'il couvre, traduisant une relation tripartite entre l'homme, les plantes et la zone écologique. Par conséquent, les plantes retrouvées dans une zone écologique sont considérées comme le patrimoine des peuples qui y sont installés et les connaissances liées à elles leurs sont caractéristiques (Dassou et al., 2015). En tenant compte de l'origine des connaissances ethnobotaniques des plantes, l'héritage et les connaissances issues des écoles de médecine traditionnelle sont des facteurs favorables dont dépendent les connaissances ethnobotaniques. En effet, selon Upadhyay et al. (2011), les recettes dans le domaine de la pharmacopée traditionnelle sont transmises oralement de génération en génération au sein de la société et demeurent un patrimoine soit de la famille, soit d'un groupe social particulier du village ou de la contrée. Pour ce fait, la connaissance des usages des plantes médicinales et leurs propriétés sont généralement acquises suite à une longue expérience accumulée et transmise d'une génération à l'autre, par voie de l'oralité.

L'expérience accumulée avec l'âge constitue donc la principale source d'information à l'échelle locale au sujet de l'usage des plantes en médecine traditionnelle (Mehdioui et Kahouadji, 2007). Cela suppose que la connaissance des plantes par un individu serait alors fonction de la réputation par rapport à la médicine traditionnelle de la communauté dans laquelle il vit ou de celle de son appartenance à un groupe ethnique. Ce qui dénote de la transmission verticale des connaissances endogènes au sein des communautés. De plus, lorsque l'individu fréquente une école de médecine traditionnelle, il pourra capitaliser en plus de ces connaissances endogènes, celles issues de sa formation en médecine traditionnelle. Ce qui lui permettra d'avoir une connaissance plus approfondie sur le traitement des maladies par les plantes.

\section{Conclusion}

Les populations au Sud du Bénin utilisent les plantes pour les soins de santé des enfants. La connaissance ethnobotanique des recettes de plantes traitant les maladies infantiles est influencée par l'appartenance à un groupe socioculturel, la spécialisation de l'individu dans la médicine traditionnelle et l'origine de la connaissance. Lorsqu'on considère l'appartenance à un groupe socioculturel, les Fon et les Goun ont une meilleure connaissance des plantes que les autres groupes alors les Holli maîtrisent mieux la connaissance des recettes. En tenant compte de la spécialisation de l'individu dans la médicine traditionnelle, les vendeurs et vendeuses de plantes et les personnes ayant une formation en médicine traditionnelle connaissent plus de plantes et de recettes. La transmission des connaissances sur les plantes traitant les maladies infantiles se fait de façon verticale au sein d'une communauté, d'une famille ou d'un groupe socioculturel. Les individus utilisent les espèces végétales retrouvées dans leur zone écologique (district phytogéographique) pour soigner les enfants. De plus, en fonction de l'appartenance à un district phytogéographique, les individus résidant dans les districts phytogéographiques Zou et Côtier ont une connaissance plus accentuée que ceux appartenant aux autres zones.

\section{CONFLIT D'INTERETS}

Les auteurs de ce manuscrit déclarent qu'il n'y a aucun conflit d'intérêt.

\section{CONTRIBUTIONS DES AUTEURS}

ASK a assuré la conception de la méthodologie, la collecte et le traitement des données puis la rédaction du manuscrit. Les autres co-auteurs ont contribué à la relecture de la méthodologie et des différentes versions du manuscrit pour l'amélioration de sa qualité scientifique. 


\section{REMERCIEMENTS}

Les auteurs remercient sincèrement les populations au Sud du Bénin pour leur disponibilité et leur franche collaboration lors de la collecte des données de terrain.

\section{REFERENCE}

Adomou AC, Agbani OP, Sinsin B. 2011. Plantes. In Protection de la Nature enAfrique de l'Ouest: Une Liste Rougepour le Bénin. Nature Conservation inWest Africa: Red List for Benin, Neuenschwander P, Sinsin B, Goergen $G$ (eds). International Institute of Tropical Agriculture: Ibadan; Nigeria; 21-46.

Akoègninou A. 2004. Recherches botaniques et écologiques sur les forêts actuelles du Bénin. Thèse d'Etat. Université de Cocody-Abidjan (Côte d'Ivoire),p.326

Akoègninou $\mathrm{A}$, van der Burg $\mathrm{WJ}$, van der Maesen LJG. 2006. Flore Analytique duBénin. Backhuys Publishers: Wageningen; 1034.

Assogbadjo AE, GlèlèKakaï R, Adjallala FH, AzihouAF, VodouhêGF, KyndtT, JTC Codjia. 2011. Ethnic differences in use value and use patterns of the threatened multipurpose scrambling shrub (Caesalpinia bonduc L.) in Benin. Journal of Medicinal Plants Research,5: 1549-1557.

Badiane SD, Mbaye E, Coly A, Kane A.2015. « Gnei-gnei» et pharmacopée traditionnelle en Basse Casamance (Sénégal): Leçons d'investigations sur le sacré et la biodiversité. Liens Nouvelle Série. $\mathrm{N}^{\circ} 20$.

Banerjee H, Goswami R, Chakraborty S, Dutta S, Majumdar K, Satyanarayana T, Jat MI, Zingore S. 2014. Understanding biophysical and socio-economic determinants of maize (Zea mays L.) yield variability in eastern India. NJASWageningen Journal of Life Sciences, 70-71: 79-93.

Beltrán-Rodríguez L, Ortiz-Sánchez A, Mariano NA, Maldonado-Almanza B and Reyes-García V.2014.Factors affecting ethnobotanical knowledge in a mestizo community of the Sierra de Huautla Biosphere Reserve, MexicoJournal of Ethnobiology and Ethnomedicine, 10: 2-18.

Benkhnigue O, Zidane L, Fadli M, Elyacoubi H, Rochdi Aet Douira A. 2011. Etudeethnobotanique des plantes médicinales dans la région de Mechraâ Bel Ksiri (Région du Gharb du Maroc). Acta Bot. Barc.,53: 191-216.

Byg A, Balslev H. 2004. Factors affecting local knowledge of palms in Nangaritza Valley, Southeastern Ecuador. Journal of Ethn, 24(2): 255-278.

Dassou HG, Ogni CA, Yedomonhan H, Adomou AC, Tossou M, Dougnon JT, AkoegninouA. 2014. Diversité usage vétérinaire et vulnérabilité des plantes médecinales au Nord-Bénin. Int. J. Biol. Chem. Sci., 8(1): 189-210. DOI: http://dx.doi.org/10.4314/ijbcs.v8i1.18

Dassou HG, Yedomonhan H, Adomou AC, Ogni CA, Tossou MG, Akoegninou A. 2015. Facteurs socioculturels et environnementaux déterminant la connaissance ethno vétérinaire au Bénin. Afrique SCIENCE, 11(5): 335-360. http://www.afriquescience.info

DonouHounsode MT, Assogbadjo AE, Houehanou T, GlèlèKakaï RL, Agbangla C. 2016. Facteurs socioéconomiques influençant l'usage des Raphias au Bénin (Afrique de l'Ouest). Science de la Vie, de la Terre et AgronomieREV. CAMES, 4(1): 2424-7235.

Gouwakinnou GN, Lykke AM, Assogbadjo AE, Sinsin B. 2011.Local knowledge, patternand diversity of use of Sclerocaryabirrea.Journal of Ethnobiology and Ethnomedicine, 7(8): 1746-4269.

Igue AM, Saidou A, Adjanohoun A, Ezui G, Attiogbe P, Kpagbin G, GotoechanHodonou H, Youl S, Pare T, Balogoun I, Ouedraogo J, Dossa E,Mando A,Sogbedji JM. 2013. Evaluation de la fertilité des sols au sud et centre du Bénin. Bulletin de la Recherche 
Agronomique du Bénin (BRAB). Disponible en ligne sur le site web http://www.slire.net

INSAE (Institut National de la Statistique et de l'Analyse économique). 2013. Recensement Général de la Population et de l'Habitat. Résultats Provisoires du RGPH4, MDAEP. INSAE: Cotonou, Bénin; 8p.

Kouchadé AS, Adomou AC, Tossou GM, Yédomonhan $\mathrm{H}$, Dassou $\mathrm{GH}$ et Akoègninou A.2016. Étude ethnobotanique des plantes médicinales utilisées dans le traitement des maladies infantiles et vendues sur les marchés au sud du Bénin. Journal of Animal \& Plant Sciences, 28(2): 4418-4438. Disponible en ligne sur http://www.m.elewa.org/JAPS;

Lougbegnon TO, Tente BAH, Amontcha M et Codjia JTC. 2011. Importance culturelle et valeur d'usage des ressources végétales de la réserve forestière marécageuse de la vallée de Sitatunga et zones connexes. Bulletin de la Recherche Agronomique du Bénin. Numéro 70.

Mehdioui R,Kahouadji A. 2007. Etude ethnobotanique auprès de la population riveraine de la forêt d'Amsittène : cas de la Commune d'Imi n'Tlit (Province d'Essaouira). Bulletin de l'Institut Scientifique, Rabat, section Sciences de la Vie, 29: 11-20.

Ryan C, Guido P, Doel S, 2008. Factors in maintaining indigenous knowledge among ethnic communities of Manus island. Econ Bot.,59: 356-365.
Thomas E, Vandebroek I, Sanca S, Van Damme P. 2009. Cultural significance of medicinal plant families and species among Quefarmers in Apillapampa, Bolivia. J. Ethnopharmacol, 122:60-67.

Turner NJ, Turner KL 2008. Where women used to get the food: cumulative effects and loss of ethnobotanical knowledge and practice; case study from coastal British Columbia. NCR Research Press, 86: 103-115.

Traoré M. 2015. Le Recours à la Pharmacopée Traditionnelle Africaine dans leNouveau Millénaire : «Cas des Femmes Herboristes de Bamako» Référence Prélude : HT 32. Disponible en ligne sur http://www.codesria.org/Archives/ga10/ Abstracts\%20GA\%2015/AIDS_Traore.htm.

Upadhyay B, Singh KP, Kumar A. 2011. Ethno-veterinary uses and informants consensus factor of medicinal plants of Sariska region, Rajasthan, India. Journal of Ethnopharmacology, 133: 14-25.

Yoro BM. 2012. Pluralisme thérapeutique et recours aux soins en milieu rural ivoirien 1 : approche méthodologique. Recherches Qualitatives, 31(1): 4761.Disponible sur http://www.recherche qualitative.qc.ca/Revue.html (C)

Sinsin B, Kampmann D. 2010. Atlas de la biodiversité de l'Afrique de l'Ouest, Tome 1 : Cotonou \&Frankfurt/Main.

Zerbo P, Millogo-rasolodimby J, NacoulmaOuedraogo OG, Damme PV. 2011. Plantes médicinales et pratiques médicales au Burkina Faso: cas des Sanan. Bois et Forêts des Tropiques, 307: 41-53. 